\title{
WORKFORCE A review of the clinical assistant workforce at a district general hospital during the COVID-19 pandemic
}

\author{
Authors: Nicole L Brown, ${ }^{\mathrm{A}}$ Sebastian A Moshtael, ${ }^{\mathrm{A}}$ Michaela Rogers, ${ }^{\mathrm{B}}$ Idil Mohamed, ${ }^{\mathrm{C}}$ Ben Smith, ${ }^{\mathrm{D}}$ \\ Christopher T Rimmer, ${ }^{\mathrm{E}}$ Adeel $\mathrm{Hamad}^{\mathrm{F}}$ and Angela $\mathrm{Yan}^{\mathrm{G}}$
}

\section{Introduction \\ In April 2020, a new workforce of clinical assistants (CAs), comprising predominantly of medical students, began work at Northampton General Hospital. Clinical-years students had a role similar to final-year student assistants; pre- clinical students were offered a healthcare assistant role. This research aimed to evaluate both CAs' and clinicians' perceptions of this programme.}

\section{Methods}

Separate questionnaires were developed for CAs and clinicians, assessing the scheme's successes and failures. Data analysis was carried out using MS Excel and SPSS.

\section{Results and discussion}

Forty-nine CAs and 60 clinicians responded. CAs of all years were completing the higher-level role. They were perceived to improve continuity of care (74\% CA agreement; $88 \%$ clinician agreement), reduce clinician workload ( $90 \%$ clinician agreement) and felt significantly more confident with practical and administrative tasks. Sixty-eight per cent of CAs and $72 \%$ of clinicians believed the role should be available to students before their final year.

KEYWORDS: assistantships, preparedness for practice, continuity of care

DOI: $10.7861 /$ fhj.2021-0056

\section{Introduction}

The first wave of COVID-19 profoundly impacted medical education in the UK, with medical schools suspending teaching, placements and exams. ${ }^{1}$ Those in higher years of study, therefore,

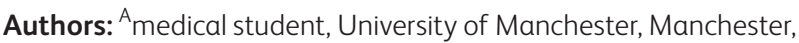
UK; ${ }^{B}$ medical student, University of Bristol, Bristol, UK; ' ${ }^{\text {medical }}$ student, University of Birmingham, Birmingham, UK; ${ }^{\text {foundation }}$ year-1 doctor, Frimley Park Hospital, Frimley, UK; E foundation year1 doctor, University Hospitals of Leicester, Leicester, UK; ${ }^{\text {F }}$ clinical education fellow in medicine, Northampton General Hospital, Northampton, UK; ${ }^{G}$ internal medicine trainee, St George's Hospital, London, UK lost valuable experience required for their careers. ${ }^{2}$ Despite uncertainty regarding their studies and the Medical Schools Council advice that education, health and safety must be prioritised, many students felt a duty to aid the country's pandemic response. ${ }^{1-3}$ Some assisted with childcare for key workers and distribution of medical supplies, while others returned to, or joined, the NHS. ${ }^{1}$

Nationwide, the NHS welcomed medical students into their workforce, offering roles such as video call provision, phlebotomy and 111 phone call operation. A select number of hospitals (including Northampton General Hospital (NGH)) took this further by using a portion of their NHS COVID-19 budget to offer a novel paid role: the clinical assistant (CA). ${ }^{4}$ Due to staffing pressures caused by the pandemic, CAs were expected to work alongside staff members, instead of simply 'being present in a ward environment' as they would during normal medical school placements. ${ }^{5}$ Pre-clinical students were to fulfil a 'lower tier' role, with responsibilities similar to those of healthcare assistants (HCA). Senior students were expected to support junior doctors with wardbased tasks as 'higher tier' CAs. This higher tier role was similar to that expected from a final year during their student assistantship. This is a General Medical Council (GMC) required placement in which a student 'under supervision undertakes most of the duties of an F1 doctor', intended to offer 'experiential learning' in order to more adequately prepare students for practice. ${ }^{5}$

The CA role attracted national attention from medical students with all levels of clinical experience, physician associate trainees and international medical graduates (IMGs) without GMC registration. The first eight CAs began work in early April 2020, and by early May, over 70 were employed.

The aim of this paper is to evaluate the CA role, identifying areas of success, improvement and learning points for future medical education by exploring both the perspectives of CAs and clinicians. Specifically, we assess support provided by NGH and the extent, if any, of the CAs' ongoing medical school commitments. We will also examine CA confidence with ward-based tasks and clinician views of CA competence as a measure of preparedness for practice. Finally, we discuss whether students and clinicians would mutually benefit from a continuation of the CA role in future medical education, or as a part-time job.

\section{Methods}

Recruitment, training and the CA role

The role was advertised in late March through a variety of methods: online (NHS jobs and social media), word of mouth 
and, for Leicester students, through direct communication from their medical school. It was an open application, working on a 'first-come first-served basis'. The deputy director of medical education and director of undergraduate studies conducted a telephone interview with each applicant to explain the role, assess their suitability for either the lower or higher tier role and answer any questions. A face-to-face induction was provided, which covered safety, clinical skills, use of IT systems and documentation. Descriptions of the two CA roles can be found in Table 1.

\section{Data collection: CAs}

The study team designed one questionnaire for CAs and one for clinicians (supplementary material S1 and S2). CAs were contacted over a 3-week period in June and July 2020 via WhatsApp or email to complete a paper or electronic questionnaire anonymously. Demographics that were collected were age, gender, university, year of study and amount of previous clinical experience. CAs also documented whether they had been trained for the HCA-style role and whether they were still completing HCA tasks at the time of data collection. If not, they were asked to state their reasons using the free-text space provided. A Likert scale (from 1 to 5) was used to rank confidence levels in performing practical procedures and other ward-based tasks prior to and after working at NGH, with an accompanying free-text space allowing CAs to document any additional new/improved skills. A 5-point Likert scale (from strongly disagree to strongly agree) assessed their experiences of working at NGH and another scale (from never to always) assessed the extent to which CAs felt part of the clinical team compared with placement at medical school, if applicable. Responses of 'always'

Table 1. Responsibilities of the lower and higher tier clinical assistant roles

\begin{tabular}{|c|c|c|}
\hline Role & Lower tier & Higher tier \\
\hline Practical tasks & $\begin{array}{l}\text { Measuring patient } \\
\text { observations } \\
\text { Changing beds } \\
\text { Personal care } \\
\text { Mouth care }\end{array}$ & $\begin{array}{l}\text { Venepuncture } \\
\text { Arterial blood gas } \\
\text { (ABG) collection } \\
\text { Venous blood gas } \\
\text { (VBG) collection } \\
\text { Cannulation } \\
\text { Catheterisation } \\
\text { Blood cultures } \\
\text { NG tube insertion }\end{array}$ \\
\hline Documentation & $\begin{array}{l}\text { NEWS } \\
\text { Food/fluid/stool } \\
\text { charts }\end{array}$ & $\begin{array}{l}\text { Preparing patient notes } \\
\text { for ward rounds } \\
\text { Scribing on ward rounds } \\
\text { Writing discharge } \\
\text { summaries (counter- } \\
\text { signed by clinicians) } \\
\text { Prescribing/rewriting } \\
\text { drug charts (counter- } \\
\text { signed by clinicians) }\end{array}$ \\
\hline Communication & & $\begin{array}{l}\text { Requesting and vetting } \\
\text { scans } \\
\text { Clerking patients } \\
\text { Updating family } \\
\text { members }\end{array}$ \\
\hline
\end{tabular}

NEWS $=$ National Early Warning Score; NG = nasogastic. or 'often' were grouped into feeling part of the clinical team and responses of 'sometimes', 'rarely' or 'never' were grouped into not feeling part of the clinical team. The same scale was used to assess continuity of care. Medical student CAs were asked about medical school commitments during their time at NGH. All CAs were also asked to estimate their attendance to formal teaching provided by $\mathrm{NGH}$, and how useful they perceived this to be. Support for CAs was assessed through tick-box questions. Finally, CAs were asked whether or not the role should be integrated into medical education. A free-text question at the end provided an opportunity for additional feedback.

\section{Data collection: clinicians}

Clinicians were approached in person over a 5 -day period in July 2020 to fill in the questionnaire. An electronic survey (via SurveyMonkey) was also distributed on a junior doctor WhatsApp group. Demographics that were collected were the clinicians' medical grades. Clinicians were asked to rank their agreement using a 5-point Likert scale (from strongly disagree to strongly agree) with statements on the CAs' impact on the clinical team, such as reducing workload. They were then asked to select from a list which skills they felt that CAs had developed. Another multiple-choice question asked whether they thought that a CA programme should be incorporated into the medical school curriculum and, if so, when, or as a part-time job. A free-text question at the end allowed for further feedback.

\section{Data analysis}

Data analysis was carried out using MS Excel and SPSS. Wilcoxon signed-rank test assessed whether CAs' confidence levels in clinical skills and ward-based tasks increased significantly during their time at NGH. Chi-squared test of independence determined whether CAs felt that they were part of the clinical team significantly more often than they did as medical students.

\section{Results}

\section{CA results}

A total of 57 questionnaires were distributed ( 28 paper and 29 electronic) and 49 were returned (response rate $=86 \%$ ). CA demographics are shown in Table 2 . The cohort consisted of predominantly third- to fifth-year medical students with 1 to 2 years' clinical experience, with level of clinical experience ranging from no experience to 4 years' experience as a doctor. The majority of CAs were UK medical students, though the role was taken up by both international medical students and graduates alike. The median number of shifts worked by CAs at the time of data collection was 27 (range 5-57), and the majority of CAs (84\%) worked day shifts. The median number of hours worked per week was 32 (range 8-50). Twenty-four per cent of CAs missed shifts after developing COVID-19 symptoms and $43 \%$ reported not having access to fit testing.

Although two separate roles were described, almost all CAs were undertaking the higher role regardless of their year of study, with only one CA who underwent HCA training still fulfilling this role at the time of data collection. Nine CAs gave reasons for this, which included adequate staffing of HCAs and nurses and feeling able to assist doctors after being offered upskilling (eg training in procedural skills). 
Table 2. Clinical assistant demographics

Gender

Female

22

Male

Age

$19-24$ years

25-29 years

30-36 years 4

Did not disclose 4

Level of medical education and role

1st- or 2nd-year medical student 12

3rd- or 4th-year medical student 23

5th-year medical student 2

Intercalating medical student 2

Pre-registration physician associate $\quad 1$

International medical graduate 8

Not disclosed

1

Self-perceived CA confidence levels in performing all practical procedures and administrative tasks significantly increased while working at NGH (Table 3). Through open-text feedback, CAs shared how they felt more confident with family discussions, prescribing, liaising with other specialties, assessing capacity, using the bleep and IT systems, and electrocardiography interpretation.

A $2 \times 2$ chi-squared test of independence assessed whether CAs felt more integrated into the medical team as a CA, or as a medical student on placement. It showed that feeling part of the clinical team was significantly associated with working as a CA (chi-squared (1; $n=39)$ 33.46; $p<0.001)$. Cramer's $V$ test revealed that feeling part of the clinical team and participant role (medical student vs $(A)$ ) shared $43 \%$ variance.

When asked whether they provided continuity of care to patients by working on the same ward or within the same specialty on a regular basis (defined as five or more shifts), $37 \%$ CAs selected 'always', 37\% selected 'often', 18\% selected 'sometimes', $4 \%$ selected 'rarely' and $4 \%$ selected 'never'.

Support for, and wellbeing of, CAs is shown in Table 4 . Of note, 10 CAs chose two sets of colleagues to whom they would escalate concerns to. The number of distressing events witnessed by CAs ranged from one to eight.

Ninety-three per cent of the 40 medical student CAs balanced this job alongside medical school commitments. Thirty-five per cent reported concurrent assessments while working at NGH, 65\% had revision for upcoming assessments, $75 \%$ had scheduled online teaching and $25 \%$ had to complete coursework or e-learning. Formal teaching was also provided for CAs by consultants at NGH. While $84 \%$ attended at least once, only $22 \%$ attended often or always, with only $43 \%$ finding the teaching consistently useful (for further data, see supplementary material S3).

Finally, $90 \%$ of medical student CAs agreed or strongly agreed that this role had been a useful learning opportunity. Seventyeight per cent agreed or strongly agreed that their experience at NGH will help them catch up with missed time at medical school due to COVID-19 and 68\% believed that clinical assistantships could be incorporated into the medical school curriculum before final year. Among the $11 \mathrm{IMGs}$ and medical students studying at medical school abroad, $64 \%$ agreed or strongly agreed that the opportunity to work as a CA would help them get a job in the NHS; the rest did not comment. Overall, $90 \%$ of CAs agreed or strongly agreed that they had enjoyed working at $\mathrm{NGH}$.

Table 3. Clinical assistant confidence levels

\section{Clinical skill}

Venepuncture

Blood cultures

ABGs

VBGs

Cannulas

Catheters

\section{Administrative task}

Prepping notes

Documenting in notes

Requesting scans

Vetting scans

Writing EDNs

Assessing an unwell

patient

Clerking: history and $\quad 44$

examination

Clerking: diagnosis and $\quad 44$

47

47

45

45

46

47 management

\section{Number of responses analysed $^{a}$}

47
42
42
43
43
36

$(1-5)$

$3(1-5)$

$2(1-5)$

$3(1-5)$

$2(1-5)$

$2(1-5)$

$2(1-5)$

$3(1-5)$

$1(1-5)$

$1(1-5)$

$1(1-5)$

$2(1-5)$

$3(1-5)$

$3(1-5)$

"Clinical assistants who had not been taught the skill, and therefor

EDNs = electronic discharge notes; $\mathrm{VBG}=$ venous blood gas. 
Table 4. Clinical assistant support and wellbeing

\begin{tabular}{ll} 
Feeling supported by the clinical team & 27 \\
Always & 18 \\
Often & 4 \\
Sometimes & 0 \\
Rarely & 0 \\
Never & \\
Escalating concerns to colleagues & 31 \\
Junior doctor & 13 \\
Consultant & 6 \\
Clinical assistant recruitment team & 2 \\
Fellow clinical assistant & 1 \\
Nurses & 4 \\
Did not know & 1 \\
Did not disclose & \\
Support service contact & 31 \\
Did know how to access & 16 \\
Did not know how to access & 2 \\
Did not disclose & \\
Distressing events witnessed & \\
Yes & 17 \\
Debrief received & 4 \\
No & 32 \\
\hline An unsuccessful cardiac arrest was the example of a distressing event \\
provided in the questionnaire.
\end{tabular}

\section{Clinician results}

Sixty questionnaires from clinicians were received. The majority of respondents were junior doctors: $28 \%$ foundation year $1,10 \%$ foundation year 2, 2\% foundation year 3, $40 \%$ core trainees and $10 \%$ registrars; $8 \%$ were consultants. The remainder did not specify their level of training. There were 316 consultants and 388 junior doctors working at NGH in July 2020; our responses represented $2 \%$ of the consultant workforce and $14 \%$ of the junior doctor workforce.

Ninety per cent of clinicians agreed or strongly agreed that CAs helped to reduce workload; $88 \%$ agreed or strongly agreed that CAs helped provide continuity of care; and $98 \%$ agreed or strongly agreed that CAs were colleagues and key members of the clinical team.

The majority of clinicians were able to offer informal teaching to CAs on the wards. Notably, $78 \%$ offered teaching on practical procedures, $75 \%$ on common medical and surgical conditions and $77 \%$ on data interpretation. More details can be found in supplementary material S3.

Several questions assessed whether clinicians perceived the programme to be beneficial to CAs. Almost all clinicians believed that CAs had developed or improved numerous skills during their time at NGH (Fig 1) and overall, $88 \%$ of clinicians believed that working as a CA will help students become more competent future doctors. Many wanted to see a continuation of the CA role; their views on how this may be implemented are shown in Fig 2 .

\section{Discussion}

Our results have demonstrated the benefits of the NGH CA programme for the CAs themselves, the clinicians they worked with and, potentially, the patients in their care. They have also highlighted important areas of improvement, should such a programme be implemented again in the future.

The first notable benefit of this programme was the potential to improve medical students' preparedness for practice. CAs showed significant improvements in confidence with practical procedures and ward-based administrative tasks after working at $\mathrm{NGH}$. These results may have been expected, as confidence levels are known to improve post-intervention. However, clinicians also believed that CAs had become more competent in performing practical skills, as well as developing their communication skills, understanding of the junior doctor role and of how the NHS works. These results are consistent with literature evaluating final-year student assistantships, a placement following the medical apprenticeship model, in which the focus is on learning through participation and involving medical students in practice as much as possible. ${ }^{6}$ A review of the student assistantship at Queen's University Belfast found that it significantly improved students' perceptions of preparedness in almost all clinical and practical skills; improved communication with patients, relatives and colleagues; and helped students' understanding of the work environment. ${ }^{7}$ Similar results have been found at the University of Bristol; students reported increased confidence in practical, communication and documentation skills, assessing and managing patients, prescribing and understanding the roles of staff members following their assistantship. ${ }^{8}$ When discussing why their assistantship had improved confidence and preparedness, students at The University of Sheffield Medical School discussed the importance of having a sense of responsibility and a clear role. ${ }^{9}$ This is unlike normal medical school placements, in which the role of a medical student is undefined, with students feeling like a



Clinicians who believed that clinical assistants had improved or developed the competency, $\%$
Fig 1. Clinicians' views on clinical assistant improvement or development of certain competencies. 


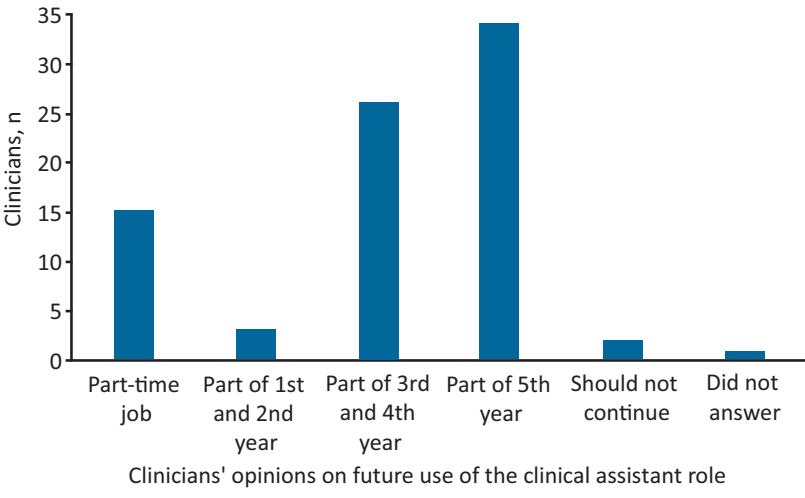

Fig 2. Clinicians' views on future applications of the clinical assistant role.

'spare wheel' and therefore adopting an overly passive approach to learning. ${ }^{10,11}$ Much like final-year assistantships, CAs at NGH were provided with specific responsibilities and expectations, perhaps aiding the shift from passive observer to active team member, and therefore providing CAs with many opportunities to practise practical and administrative skills. It is unclear whether pay was somewhat accountable for CA engagement, but the fact that similar results were found from unpaid final-year assistantships suggests that this is unlikely.

Despite significant improvements in preparedness since the introduction of the student assistantship, 33\% of UK graduates in 2019 still did not feel prepared for practice, leading to calls for longer periods of 'hands-on' experience. ${ }^{12,13}$ Assistantships are currently only offered to final-year students, but our results suggest that medical students of all years can fulfil this role, and making this available to students in earlier years of study would provide them with the practical experience they need. The majority of respondents in this study agreed with this proposal; $68 \%$ of medical student CAs and $47 \%$ of clinicians wanted to see the integration of a CA role into the first- to fourth-year medical school curriculum, and an additional $25 \%$ of clinicians wanted the CA role to become a part-time job for medical students. Overall, it is clear that the CA role helped students prepare for many of tasks expected of them when they qualify, and that prolonged exposure to such experience, whether in an unpaid medical student placement or in a paid NHS trust role, is desired by both students and clinicians.

The active engagement of CAs seemed to positively impact clinicians too, with $90 \%$ of those surveyed reporting reduced workloads, despite staffing pressure caused by the pandemic. Even before COVID-19, the strains placed upon NHS junior doctors were well documented. The 2019 national training survey revealed that over $45 \%$ of trainees regularly worked over their contracted hours, and almost $40 \%$ reported excessive workloads. ${ }^{14}$ These pressures have been shown to negatively impact patient care and clinician wellbeing, and are only set to increase as the NHS attempts to deal with the growing backlog of work (such as elective surgeries) caused by the pandemic. ${ }^{15,16}$ There may, therefore, be a role for CAs within the NHS even as COVID-19 cases start to fall. The ability of CAs to reduce clinician workload may be why they were regarded as valuable colleagues by almost all clinicians. CAs, in turn, felt significantly more integrated into the clinical team when they worked at NGH, compared with when they were medical students on placement. This is consistent with other studies, in which medical students reported feeling like anonymous bystanders during their clinical placements, while students completing their final-year assistantships reported feeling both professionally and socially accepted by colleagues. ${ }^{11,17}$ Teamwork is a cornerstone of the NHS, linked to improved patient outcomes, productivity and mental wellbeing of healthcare professionals, as well as being a GMC requirement for medical students. ${ }^{18,19}$ Hence, integration into clinical teams should be a key aspect of medical education and, unlike traditional medical school placements, the CA role has shown to be an effective way to do this.

As well as helping clinicians, CAs were also perceived to benefit patients by providing continuity of care. Like many trusts, junior doctors at NGH were redeployed to COVID-19 rotas during the pandemic, sometimes covering different wards on a daily basis. ${ }^{20,21}$ In contrast, CAs tended to work on the same wards. Continuity of care has been linked to improved clinician-patient relationships, more efficient management of patients and reduced mortality rates, again demonstrating the multiple beneficiaries of this programme. ${ }^{22}$

The concept of students spending additional hours on the wards (either as part of their placement or as a job outside of medical school hours) may lead to questions about time for academic study. Although $93 \%$ of the medical student CAs were able to balance at least one academic commitment during their time at NGH, the possibility that CAs were studying for fewer hours per week than they would at medical school cannot be excluded. However, the vast majority of clinicians were able to provide informal teaching to CAs on a regular basis, and almost $80 \%$ of CAs felt as though their assistantship made up for lost time at medical school, suggesting that this programme did provide some opportunity for CAs to learn. Moreover, $52 \%$ of medical students already manage studies alongside a part-time job in order to finance their degree, so allowing this job to complement their studies could help to promote equality among medical students and encourage widened participation within medicine. ${ }^{23}$ Introducing a new paid role within the NHS would, of course, require funding, and whether this would come from medical schools or NHS trusts needs to be addressed.

The most important area for improvement was CA access to support. The pandemic brought added stressors to doctors, and had a significant effect on their mental health. ${ }^{24}$ CAs were not exempt from this: $24 \%$ self-isolated with COVID-19 symptoms, $35 \%$ witnessed traumatic events and some worked up to 50 hours per week. Although CAs felt able to access support from colleagues, a third were unaware of how to access the hospital's support service. Even without the pressures of COVID-19, the poor mental health of doctors and medical students is well known. ${ }^{25}$

Therefore, for a workforce that was potentially naive to the mental impact of working in the NHS, let alone during a pandemic, access to support was essential. Future CA programmes should provide an easily accessible, well-advertised support service, with a cap on the number of hours a CA can work. Another aspect for improvement was formal teaching. Although a high proportion of CAs were able to attend at some point during their time at $\mathrm{NGH}$, few attended regularly. Perhaps this was due to the fact that only $60 \%$ found the teaching consistently useful, or perhaps they felt unable to leave their ward duties. This could be improved by providing protected teaching time for CAs, and by allowing them to request certain teaching topics. 
Our study's limitations include our small sample size, limited to one district general hospital, the potential for recall bias (as some CAs were no longer working at NGH at time of data collection), the use of self-assessed confidence instead of objective competence to measure practical and administered competence, and that not all respondents answered all the questions. However, our accessible questionnaires resulted in a high CA response rate. Our clinician data collection, despite being small, consisted of clinicians at every medical grade who had worked alongside CAs and represented our target population. Additionally, supplementing the CA data with input from clinicians allowed us to more reliably assess the improvement in CAs' skills levels.

\section{Conclusion}

While this CA programme was initially set up to reduce strain on doctors, which seems to have been successful, it has also provided other significant benefits in terms of patient care and CA preparedness to perform many of the tasks expected of a junior doctor. Both students and clinicians want to see prolonged access to a CA-type role, whether as part of the medical school curriculum or as a part-time job. However, issues such as funding and practical implementation alongside academic study would need to be addressed, as would CA access to formal support services.

\section{Supplementary material}

Additional supplementary material may be found in the online version of this article at www.rcpjournals.org/fhj:

S1 - Questionnaire for clinical assistants.

S2 - Questionnaire for clinicians.

S3 - Formal and informal teaching opportunities.

\section{References}

1 Kinder F, Harvey A. Covid-19: the medical students responding to the pandemic. BMJ 2020;369:m2160.

2 Al Samaraee A. The impact of the COVID-19 pandemic on medical education. Br J Hosp Med 2020;81:1-4.

3 Medical Schools Council. Statement of expectation. MSC, 2020. www.medschools.ac.uk/media/2641/updated-volunteeringguidance-020420.pdf

4 Wilson LA, Bowie J, Jewell M, O'Donnell A, Abesinghe N. Rapid response to Covid-19: the medical students responding to the pandemic. BMJ 2020;369:m2160.

5 General Medical Council. Clinical placements for medical students. GMC, 2011. www.gmc-uk.org/-/media/documents/Clinical_placements_for_medical_students__guidance_0815.pdf_56437824. pdf

6 Kopelman P. The future of UK medical education curriculum - what type of medical graduates do we need? FHJ 2014:1:41-6.

7 Braniff C, Spence RA, Stevenson M, Boohan M, Watson P. Assistantship improves medical students' perception of their preparedness for starting work. Med Teach 2016;38:51-8.

8 Hawkins A, Stanton A, Forbes K. An extended assistantship for finalyear students. Clin Teach 2015;12:305-9.

9 Lightman E, Kingdon S, Nelson M. A prolonged assistantship for final-year students. Clin Teach 2015;12:115-20.
10 Crossley JG, Vivekananda-Schmidt P. Student assistantships: bridging the gap between student and doctor. Adv Med Educ Pract 2015:6:447-57.

11 Dornan T, Boshuizen H, King N, Scherpbier A. Experience-based learning: a model linking the processes and outcomes of medical students' workplace learning. Med Educ 2007:41:84-91.

12 General Medical Council. Be prepared: are new doctors safe to practise? GMC, 2014. https://prescribingsafetyassessment.ac.uk/ resources/be_prepared.pdf

13 Dornan T, Gillespie H, Armour D, Reid H, Bennett D. Medical students need experience not just competence. BMJ 2020; 371:m4298.

14 General Medical Council. National training surveys 2019: Initial findings report. GMC, 2019. www.gmc-uk.org/-/media/documents/ national-training-surveys-initial-findings-report-2019_pdf84390391.pdf

15 British Medical Association. Fatigue and sleep deprivation - the impact of different working patterns on doctors. BMA, 2018. www. bma.org.uk/media/1074/bma_fatigue-sleep-deprivation-briefingjan2017.pdf

16 Mahase E. NHS and social care need an extra $€ 12 \mathrm{bn}$ to get back on track after pandemic, says think tank. BMJ 2021;372:n721.

17 Jones OM, Okeke C, Bullock A, Wells SE, Monrouxe LV. 'He's going to be a doctor in August': a narrative interview study of medical students' and their educators' experiences of aligned and misaligned assistantships. BMJ Open 2016;6:e011817.

18 Chandrashekar A, Mohan J. Preparing for the National Health Service: the importance of teamwork training in the United Kingdom medical school curriculum. Adv Med Educ Pract 2019;10:679-88.

19 General Medical Council. Outcomes for graduates. GMC, 2018. www.gmc-uk.org/-/media/documents/dc11326-outcomes-forgraduates-2018_pdf-75040796.pdf

20 Joseph AO, Joseph JP, Gahir J, Pereira B. Re-organising junior doc tors during the COVID-19 Outbreak: a single centre experience in the United Kingdom. Int J Health Policy Manag 2020;9:459-60.

21 Jeyabaladevan P. COVID-19: an FY1 on the frontline. Med Educ Online 2020;25:1759869.

22 Pereira Gray DJ, Sidaway-Lee K, White E, Thorne A, Evans PH. Continuity of care with doctors-a matter of life and death? A systematic review of continuity of care and mortality. BMJ Open 2018:8:e021161.

23 Royal Medical Benevolent Fund. Our medical student survey findings. Royal Medical Benevolent Fund, 2018. https://rmbf.org/about/ our-campaigns/medstudentsmatter/our-medical-student-surveyfindings [Accessed 13 February 2021].

24 Shaukat N, Ali DM, Razzak J. Physical and mental health impacts of COVID-19 on healthcare workers: a scoping review. Int J Emerg Med 2020;13:40.

25 Bhugra D, Sauerteig S-O, Bland D et al A descriptive study of mental health and wellbeing of doctors and medical students in the UK. Int Rev Psychiatry 2019;31:563-8.

Address for correspondence: Ms Nicole Brown, Faculty of Biology, Medicine and Health, The University of Manchester, Oxford Road, Manchester M13 9PL, UK. Email: nicole.brown@student.manchester.ac.uk 